\title{
Prefrontal-Inferotemporal Interaction Is Not Always Necessary for Reversal Learning
}

\author{
Charles R. E. Wilson and David Gaffan \\ Department of Experimental Psychology, Oxford University, Oxford OX1 3UD, United Kingdom
}

Prefrontal cortex (PFC) is thought to have a wide-ranging role in cognition, often described as executive function or behavioral inhibition. A specific example of such a role is the inhibition of representations in more posterior regions of cortex in a "top-down" manner, a function thought to be tested by reversal learning tasks. The direct action of PFC on posterior regions can be directly tested by disconnecting PFC from the region in question. We tested whether PFC directly inhibits visual object representations in inferotemporal cortex (IT) during reversal learning by studying the effect, in macaque monkeys, of disconnecting PFC from IT by crossed unilateral ablations. We tested two visual object reversal learning tasks, namely serial and concurrent reversal learning. We found that the disconnection severely impairs serial reversal learning but leaves concurrent reversal learning completely intact. Thus, PFC cannot be said to always have direct inhibitory control over visual object representations in reversal learning. Furthermore, our results cannot be explained by generalized theories of PFC function such as executive function and behavioral inhibition, because those theories do not make predictions that differentiate different forms of reversal learning. The results do, however, support our proposal, based on other experimental evidence from macaque monkeys, that $\mathrm{PFC}$ has a highly specific role in the representation of temporally complex events.

Key words: monkey; reversal learning; PFC; IT; executive function; inhibition; macaque; memory; prefrontal

\section{Introduction}

Prefrontal-inferotemporal disconnection in monkeys can be accomplished by crossed unilateral ablations of inferotemporal cortex (IT) in one hemisphere and of prefrontal cortex (PFC) in the other hemisphere (PFCxIT) (Fig. 1). Because the connections between these cortical areas are predominantly within each hemisphere (Kuypers et al., 1965; Jones and Powell, 1970), PFCxIT has the effect of disconnecting them from each other but leaving the animal with functional areas of both PFC and IT. The disconnection procedure therefore allows us to specifically investigate whether PFC acts directly on inferotemporal visual information in a given task.

Previous experiments have shown that PFCxIT disconnection produces strikingly different effects on different visual learning tasks in the macaque monkey. It leaves intact the monkey's ability in concurrent visual discrimination learning. In this task, the animal learns to associate visually presented objects with food reward or absence, and successive presentations of each individual object are separated in time by the concurrent presentation of other objects (Parker and Gaffan, 1998b; Gaffan et al., 2002). In

Received Dec. 9, 2007; revised April 8, 2008; accepted April 14, 2008.

This work was supported by the Medical Research Council (United Kingdom). C.R.E.W. was supported by the Christopher Welch Trust. We thank Mark G. Baxter for assistance with anesthetic procedures and both Mark G. Baxter and Philip G. F. Browning for helpful comments. We thank M. Brown, G. Daubney, K. Murphy, and S. Mygdal for technical support. C.R.E.W. and D.G. designed this study, discussed the results, and edited this manuscript together C.R.E.W. tested the monkeys and wrote this manuscript.

The authors declare no competing financial interests.

Correspondence should be addressed to Charles R. E. Wilson, Department of Experimental Psychology, 0xford University, South Parks Road, 0xford OX1 3UD, UK. E-mail: charles.wilson@psy.ox.ac.uk.

DOI:10.1523/JNEUROSCI.0952-08.2008

Copyright $\odot 2008$ Society for Neuroscience $\quad$ 0270-6474/08/285529-10\$15.00/0 contrast, the same disconnection produces a severe impairment in serial visual discrimination learning, in which the successive trials with any individual object follow immediately on each other (Browning et al., 2007). In serial visual learning, but not in concurrent visual learning, monkeys form an integrated representation of successive trials with the same object, allowing a rapid rate of within-problem learning to develop with learning set formation (Murray and Gaffan, 2006). The pattern of results from disconnection in these two tasks is therefore consistent with the general idea (Browning et al., 2005, 2007; Browning and Gaffan, 2008) that prefrontal-inferotemporal interaction is required when the monkey forms an integrated representation of a temporally complex visual event involving two or more successive trials with visual objects but not when the monkey forms a representation of a temporally simple event involving only the delivery of a food reward after a single visual presentation.

Impairments in reversal learning are a key component of the evidence for a putative broad inhibitory role of PFC in humans (Milner, 1963; Rolls et al., 1994; Konishi et al., 1999; Fellows and Farah, 2003; Aron et al., 2004) and animals (Brutkowski, 1965; Iversen and Mishkin, 1970; Dias et al., 1997; Nakahara et al., 2002; Clarke et al., 2004; Izquierdo et al., 2004). However, the temporal complexity theory would predict that PFCxIT should impair reversal learning only if the task involves the processing of temporally complex visual events. Just as in discrimination learning, so also in reversal learning, PFCxIT should impair serial reversal learning, which promotes reversal learning set but not concurrent reversal learning. To test this prediction, we tested the effect of PFCxIT on serial and concurrent reversal learning. Experiment 1 investigated concurrent reversal learning, and experiment 2 investigated serial reversal learning. 


\section{Materials and Methods}

Subjects

In experiment 1 , the subjects were six male cynomolgus monkeys (Macaca fascicularis) of mean age 3 years and 6 months and mean weight $5.3 \mathrm{~kg}$, at the start of the present experiment. Before beginning the present experiment, they had performed a series of experiments on concurrent visual discrimination learning (Browning and Gaffan, 2008). All six monkeys had the same behavioral training history, and this had not included reversal learning. In the course of their previous experiments, three of these monkeys had received PFC-IT disconnection. The surgical operations to perform this disconnection, described below, took place 383-413 $\mathrm{d}$ before the present experiment began. In experiment 2 , the subjects were six male rhesus monkeys (Macaca mulatta) of mean age 3 years and 0 months and of mean weight $3.9 \mathrm{~kg}$ at the start of testing. These animals had acted previously as subjects in experiments involving memory for visual stimuli (Mitchell and Gaffan, 2008) but not involving any form of reversal learning, and all had the same training history. As part of their training, all 12 monkeys learned to respond to images in the automated apparatus (see below) and to take food rewards. The two species of macaque monkey have been used interchangeably in macaque research, with very few differences noted between the two, and indeed have been used together in a number of previous studies from our laboratory without showing any species effects in visual learning (Gaffan, 1994; Gaffan et al., 2002; Wilson et al., 2007). Each group of monkeys were housed as a group in a large enclosure, and water was provided ad libitum in the home enclosure.

In both experiments, monkeys were matched on preoperative behavioral ability and separated into lesion and unoperated control groups (in each case, $n=3$ ). In experiment 1 , the lesion group had received a disconnection of prefrontal cortex and inferotemporal cortex (PFCxIT) before the commencement of the present experiment. In experiment 2 , the lesion group received PFCxIT as part of the procedure of the experiment and as described below. Within each experiment, the training history of the control and lesion groups before the present experiments was identical.

\section{Apparatus}

The apparatus consisted of a computer-controlled touch-sensitive monitor, $380 \mathrm{~mm}$ wide $\times 280 \mathrm{~mm}$ high, on which the stimulus material was presented. The monkeys were tested in a wheeled transport cage, which was placed in a small unlit testing cubicle. The cage was placed $150 \mathrm{~mm}$ from the screen, and the monkeys made choices toward stimuli by reaching out of the cage through bars on the front and touching the screen. A closed circuit television system allowed the experimenters to watch the monkey from another room, from where the stimuli, food rewards, responses, and any other experimental contingencies were all controlled automatically by computer. Rewards for correct responses consisted of a mixture of banana and sucrose pellets (190 mg; Noyes Company), and these were delivered to a small hopper situated centrally below the monitor, accompanied by an audible "click." A single large food reward was delivered at the end of each session by the automated opening of a lunchbox situated below and to the left of the screen. This large food reward comprised proprietary monkey food, fruit, peanuts, seeds, and dates. The amount of this large reward was adjusted for individuals to avoid obesity. This large food reward, together with the pellets and a small amount of forage material in the home enclosure, provided the majority of the daily diet of the monkeys on testing days.

\section{Stimulus material}

The stimuli used in both experiments were novel clipart "objects," $128 \times$ 128 pixels in size. Each object used a maximum of 20 colors and was
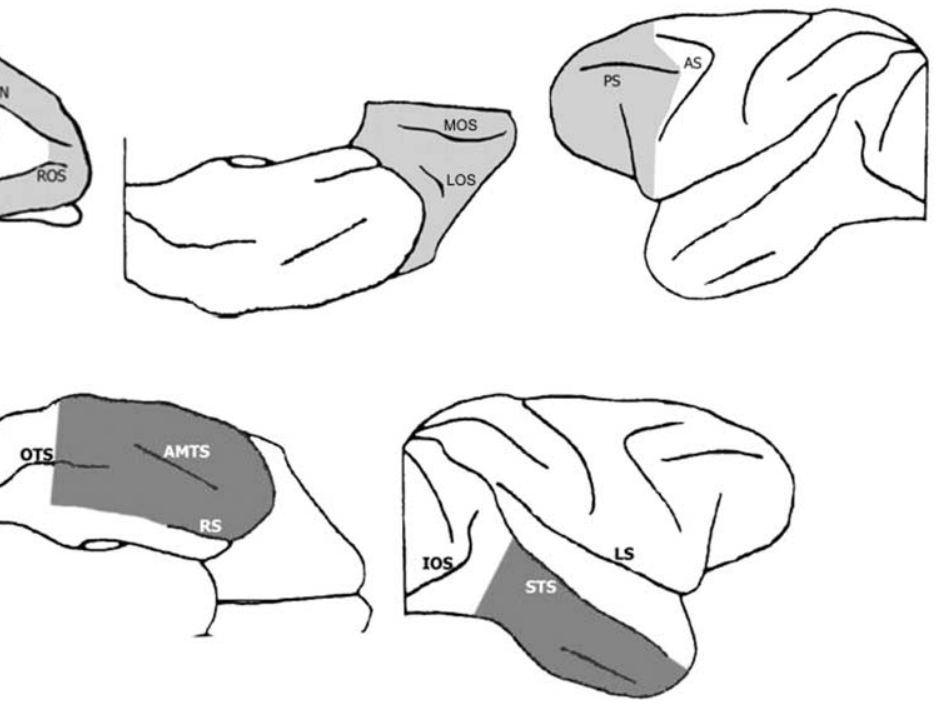

Figure 1. Intended extents of the crossed unilateral ablations of prefrontal cortex (top row, light gray) and inferotempora cortex (bottom row, dark gray) shown from medial, ventral, and lateral views. The shaded areas indicate the areas of intended removal. For detailed description, see Materials and Methods. CIN, Cingulate sulcus; ROS, rostral sulcus; LOS, lateral orbital sulcus; MOS, medial orbital sulcus; PS, principal sulcus; AS, arcuate sulcus; OTS, occipitotemporal sulcus; AMTS, anterior middle temporal sulcus; RS, rhinal sulcus; IOS, intraoccipital sulcus; STS, superior temporal sulcus; LS, lateral sulcus.

presented on a white background. These stimuli contained multiple visual elements and multiple colors, and discrimination within this set is not possible on the basis of a single feature. This means that processing within IT would be required to discriminate between them. In experiment 1, 20 objects were chosen at random from a pool of 6100 unique objects, after which an additional check was made to ensure that none of the objects were visually identical to each other, nor that discrimination was not possible on the basis of single features alone. The two stimuli that were used throughout the serial reversal experiment (experiment 2 ) were clipart stimuli from the same source as the multiple stimuli used in the concurrent reversal experiment. However, the two stimuli in the serial reversal experiment were not chosen at random. Gross (1978) and Gaffan et al. (1986) showed that monkeys with bilateral inferior temporal ablations were unimpaired in learning single visual discrimination problems when the two stimuli to be discriminated differed only in the spatial features of size or orientation (Gross, 1978; Gaffan et al., 1986); discrimination between stimuli that differ only in these spatial characteristics is dependent on parietal cortex (Eacott and Gaffan, 1991). Therefore, the two stimuli in the present serial reversal experiment were selected so as to differ from each other in visual features other than these spatial, parietaldependent features.

\section{Task procedure}

Shared aspects of the two tasks. In both tasks, the procedure of a single trial was identical, and any given trial represented an object reward discrimination between two clipart objects. At the start of a trial, two stimuli were displayed on the left and right of the screen, on the vertical midline, and equidistant from the center point. Any such pair of stimuli constituted a problem, in which one image was rewarded and one was not rewarded. The left-right position of these stimuli was determined at random for each trial. The monkey made a choice by touching the screen within the $128 \times 128$ pixel area of one of the stimuli. A correct response elicited immediate visual feedback, comprising the presentation of just the rewarded stimulus on the screen for $1 \mathrm{~s}$, as well as an immediate reward pellet or, if the response was the final correct response of a session, the large food reward (see apparatus above). An incorrect response elicited no feedback, and the screen was immediately blanked of stimuli. No reward pellet was dispensed. Trials were separated by an intertrial interval of $5 \mathrm{~s}$ after a correct choice and $10 \mathrm{~s}$ after an incorrect choice. Any touch to the screen during this intertrial interval reset the interval.

Experiment 1. Throughout the experiment, discriminations were learned concurrently in sets of 10 problems. Each presentation of the 10 
a.

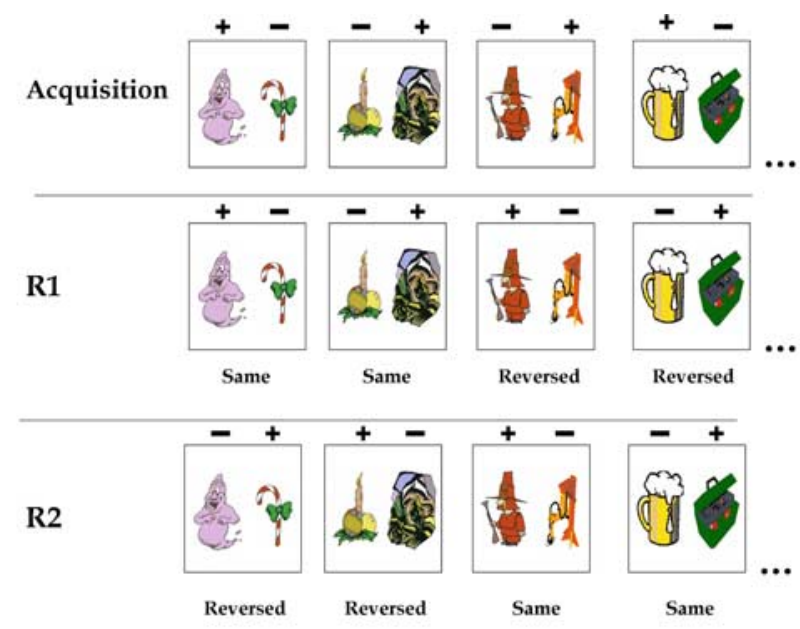

b.
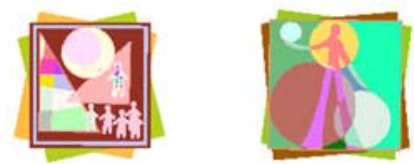

Figure 2. Stimuli from the two experiments. $\boldsymbol{a}$, Examples of stimuli and their changing reward contingencies in experiment 1 . For additional detail, see Materials and Methods, Task Procedure, Experiment 1. Each box represents two stimuli comprising a trial, presented together on the screen. There were 10 such problems in the experiment, of which four examples are shown here. Above the stimuli, a + symbol refers to the stimulus that was rewarded in that trial, and a - symbol refers to stimulus that was not rewarded on that trial. The top line refers to the initial acquisition stage, whereas subsequent lines refer to stages R1 and R2. In each case, monkeys learned a stage to a criterion of $90 \%$ within a session before moving on to the next stage. $\boldsymbol{b}$, Stimuli used in experiment 2. For details, see Materials and Methods, Task Procedures, Experiment 2. The two stimuli were the only stimuli used throughout the experiment. In any given session, one was rewarded and one was not. These contingencies reversed every time the monkey reached criterion on a session.

problems comprised a block of trials in which each of the problems was presented exactly once in an order that was randomized for each new block. A session of training consisted of 20 of these blocks and hence 200 trials. If the 200th trial was a correct response, then it opened the lunchbox revealing the large food reward. If the 200th response was incorrect, however, additional trials were presented, and the lunchbox was opened after the next correct response. Thus, the monkey only ever received the large food reward after a correct response. Monkeys continued daily sessions at one stage of training until a criterion of $90 \%$ correct choices within a session was reached, with an additional criterion that each monkey must perform a minimum of two sessions at each stage of training. The day after reaching criterion at a stage, the monkey began training on the next stage.

The experiment consisted of three stages of training. In each stage of training, the same 20 stimuli were used, used in the same 10 pairs that constituted the 10 trials in a block. The first stage was the acquisition stage (stage A), in which monkeys learned the correct responses in the 10 pairs of stimuli to criterion. The second stage was the first reversal stage (stage R1), and, at this stage, the trials consisted of the same 10 pairs of stimuli. Five of the pairs, however, had the opposite reward contingencies to the acquisition stage, in that the object that had been rewarded previously was not, whereas the previously unrewarded object was now rewarded. These trials were termed the reversed trials. The remaining five pairs retained the same contingencies as before and were termed the nonreversed trials. This ensured that no consistent rule could be applied to all of the problems with regards to reversal of reward contingencies. The reversed and nonreversed trials remained randomly ordered within each block. In a second reversal stage (stage R2), the five pairs that were nonreversed in stage R1 now had their reward contingencies reversed in a similar manner to other stimuli in stage $\mathrm{R} 1$, to become reversed trials in this stage. The five pairs that were reversed in stage $\mathrm{R} 1$ retained their reward contingencies between stages R1 and R2 and hence were nonreversed at stage R2. As such, in both stages R1 and R2, monkeys were responding to a set of 10 problems in which five had their reward contingencies reversed relative to the previous stage, and five were the same as the previous stage. Because of the pseudorandomization of the trials within each block, there was no way of knowing in advance which problems were reversed and which were not. Once the monkeys had performed stage R2 to criterion, the experiment was completed. Figure $2 a$ gives examples of stimuli and their changing reward contingencies in the task.

Experiment 2. Within each session, monkeys repeatedly received the same trial, with the same two stimuli (Fig. $2 b$ ) and the same reward contingencies. Monkeys had to select the rewarded object. There were three possible criteria for completion of the session. First, the monkey could make 30 consecutive correct responses. In this case, the last of these 30 responses opened the lunchbox revealing the large food reward. Second, the monkey could attain a total of 80 rewards within the session. In this case, the 80th correct response opened the lunchbox. In either of these two cases, the monkey was deemed to have reached criterion in that session. If the monkey did not reach criterion in the session, then he would work until he had performed 100 trials in total. At this point, the next correct response would open the lunchbox, the session would end, and criterion would not have been reached.

If monkeys reached criterion on any given testing day, then the following day they would receive the task with the object reward contingency reversed. After the very first session, each subsequent occasion of reaching criterion was counted as completion of a reversal. If, however, the monkey did not reach criterion within a session, then he would perform the task with the same contingencies as the previous day on the following day, until criterion was reached within a session.

Before surgery (or surgical breaks in the case of the controls), the monkeys were given extensive experience on the task so that they could acquire a reversal learning set. The acquisition of this learning set is shown in Figure 6. The amount of this preoperative training was governed by criteria of both duration and attainment. Monkeys had to complete 60 reversals and also complete 10 consecutive reversals making 10 or fewer errors, to move on to the surgical stage of the experiment. This ensured that the monkeys had extensive experience of the task and also a high level of performance indicative of the acquisition of a learning set. In general, monkeys achieved the performance criterion before completing the required 60 reversals.

After completion of preoperative training to both criteria, the surgical group underwent the first surgery, a unilateral ablation of prefrontal cortex (see surgery) followed by a period of recovery (mean of $22 \mathrm{~d}$ ), whereas the unoperated control group had a break from testing (mean of $19 \mathrm{~d})$. All six monkeys then completed an interim performance test comprising 12 reversals on exactly the same task as performed preoperatively. There was then a second surgery to complete the disconnection for the surgical group, with another recovery period (mean of $14 \mathrm{~d}$ ) and break from testing for the control group (mean of $17 \mathrm{~d}$ ). Finally, all six monkeys completed the final performance test, again comprising 12 reversals of the same task. After the 12th reversal of this performance test, the experiment was completed.

\section{Surgical procedures}

Neurosurgical procedures were performed in a dedicated operating theater under aseptic conditions. Intended extents of the lesions can be seen in Figure 1, and histological samples and reconstructions of the lesions can be seen in Figures 3 and 4. Neurosurgical procedures were performed 


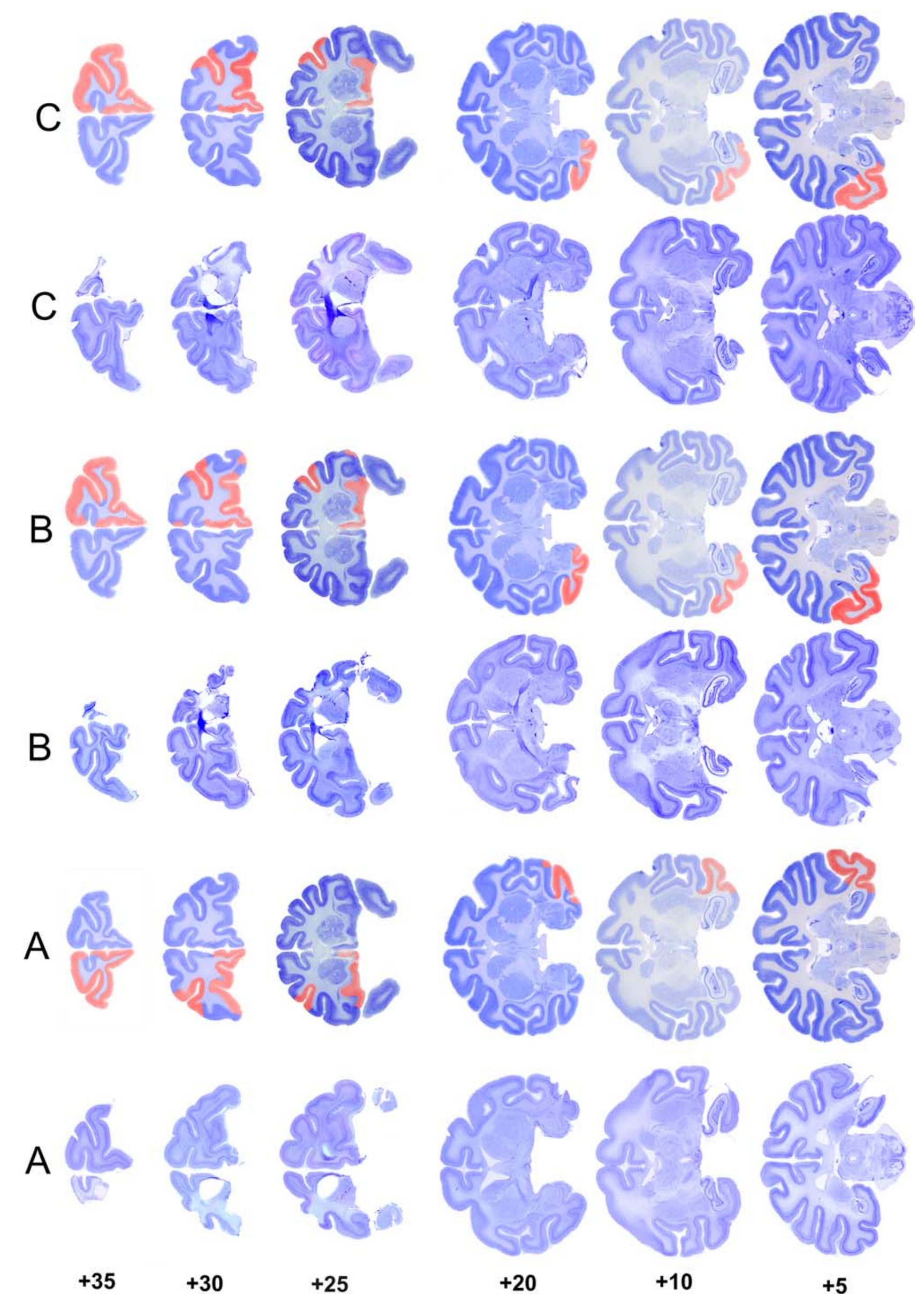


in a dedicated operating theater under aseptic conditions. During the experiment, the laboratory was undergoing changes in its anesthetic regimen under veterinary advice, and as such the anesthetic procedures in the two experiments differed slightly.

Experiment 1. Monkeys received both unilateral prefrontal and temporal lesions in a single surgical procedure.

The monkey was sedated on the morning of surgery with both ketamine $(10 \mathrm{mg} / \mathrm{kg})$ and xylazine $(0.5 \mathrm{mg} / \mathrm{kg})$ and $/$ or midazolam $(0.25$ $\mathrm{mg} / \mathrm{kg}$ ), intramuscularly. Once sedated, the monkey was given nonsteroidal anti-inflammatory (meloxicam, $0.2 \mathrm{mg} / \mathrm{kg}$, i.v.) agents for analgesia.

The head was shaved and an intravenous cannula put in place for intraoperative delivery of fluids (warmed sterile saline drip, 5 $\mathrm{ml} \cdot \mathrm{kg}^{-1} \cdot \mathrm{h}^{-1}$ ). The monkey was anesthetized throughout the surgery with barbiturate (thiopentone sodium, to effect) administered through an additional intravenous cannula. Monkeys were maintained in a state of deep anesthesia by monitoring pulse rate, blood oxygenation, body temperature, and peripheral reflexes, consistent with United Kingdom Home Office regulations.

Experiment 2. Each monkey received the disconnection in two separate surgeries, with the unilateral prefrontal ablation coming first and with a period of testing between surgeries.

Steroids (methylprednisolone, $20 \mathrm{mg} / \mathrm{kg}$ ) were given the night before surgery (intramuscularly), and three doses were given 4-6 h apart (intravenous or intramuscularly) on the day of surgery, to protect against intraoperative edema and postoperative inflammation. The monkey was sedated on the morning of surgery with both ketamine $(10 \mathrm{mg} / \mathrm{kg})$ and xylazine $(0.5 \mathrm{mg} / \mathrm{kg})$ and $/$ or midazolam $(0.25 \mathrm{mg} / \mathrm{kg})$, intramuscularly. Once sedated, the monkey was given atropine $(0.05 \mathrm{mg} / \mathrm{kg})$ to reduce secretions, antibiotic (amoxicillin, $8.75 \mathrm{mg} / \mathrm{kg}$ ) for prophylaxis of infection, opioid (buprenorphine $0.01 \mathrm{mg} / \mathrm{kg}$, i.v., repeated twice at $4-6 \mathrm{~h}$ intervals on the day of surgery, intravenously or intramuscularly) and nonsteroidal anti-inflammatory (meloxicam, $0.2 \mathrm{mg} / \mathrm{kg}$, i.v.) agents for analgesia, and an $\mathrm{H}_{2}$ receptor antagonist (ranitidine, $1 \mathrm{mg} / \mathrm{kg}$, i.v.) to protect against gastric ulceration as a side effect of the combination of steroid and nonsteroidal anti-inflammatory treatment. The head was shaved and an intravenous cannula put in place for intraoperative delivery of fluids (warmed sterile saline drip, $5 \mathrm{ml} \cdot \mathrm{kg}^{-1} \cdot \mathrm{h}^{-1}$ ). The monkey was moved into the operating theater, intubated, placed on isoflurane anesthesia (1-2.75\%, to effect, in $100 \%$ oxygen), and then mechanically ventilated. Adjustable heating blankets allowed maintenance of normal body temperature during surgery. Heart rate, oxygen saturation of hemoglobin, mean arterial blood pressure, expired $\mathrm{CO}_{2}$, body temperature, and respiration rate were monitored continuously throughout surgery.

Surgical procedures common to both experiments. After induction of anesthesia, the monkey was placed in a head holder, and the head was cleaned with alternating antimicrobial scrub and alcohol and draped to allow a midline or coronal incision. The skin and underlying galea were opened in layers. For the inferotemporal ablations, the right zygoma was removed to improve access to the temporal lobe. The temporal muscles were retracted as necessary to expose the skull surface over the intended lesion site. A bone flap was turned over the desired lesion site (prefrontal or inferotemporal), and the craniotomy was extended with rongeurs as necessary. The dura was cut and reflected over the intended lesion site. When the lesion was complete, the dura was sewn over the lesion site, and

\section{$\leftarrow$}

Figure 3. Coronal sections of the actual and reconstructed lesions of monkeys in experiment 1. This figure should be viewed in landscape format, with the two columns marked A to the left of the page. When viewed in this way, the leftmost column shows actual sections at different anteroposterior levels taken from monkey A. In the adjacent column, the extent of the cortical removal is reconstructed and shown in red on cresyl violet-stained sections taken from a normal macaque brain. The remaining columns show similar sections and reconstructions for monkeys $B$ and $C$. This method of displaying the histology better illustrates the size of the lesions, in particular the removed sulci that may not be obvious from the original sections because of collapse of overlying cortex. The numbers to the left indicate millimeters anterior to the interaural line (estimated from Paxinos et al., 2000). the bone flap was replaced and held with loose sutures. On completion of the operation, the skin and galea were closed in layers, the monkey was removed from the head holder, and anesthesia was discontinued. The monkey was returned to the home cage and monitored until normal posture was regained. Nonsteroidal anti-inflammatory analgesic (meloxicam, $0.2 \mathrm{mg} / \mathrm{kg}$, oral) and antibiotic $(8.75 \mathrm{mg} / \mathrm{kg}$, oral) treatment continued after surgery in consultation with veterinary staff, typically for $5 \mathrm{~d}$. Operated monkeys rejoined their social groups as soon as practicable after surgery, usually within $3 \mathrm{~d}$ of the operation.

Unilateral prefrontal lobe ablation. The intention was to remove prefrontal association cortex but spare cingulate cortex, supplementary, and premotor areas. A large bone flap was raised over the midline and the hemisphere to be lesioned, and the dura mater was cut. The posterior limit of the lesion on the lateral surface of the frontal lobe followed an approximate line joining the tips of the ascending and descending limbs of the arcuate sulcus and the posterior limit of the principal sulcus. The entire arcuate sulcus was to be spared. From the anteriormost point of the ascending limb of the arcuate sulcus, the boundary extended vertically and then down into the interhemispheric fissure. Medially, this boundary remained at this same anteroposterior level up to the crown of the cingulate sulcus. The entire cingulate cortex was to be spared. From the anteriormost point of the descending limb of the arcuate sulcus, the boundary extended downward to the ventral surface of the frontal lobes. All cortex forward of this boundary was removed. The entire ventral surface of the frontal lobes was removed, including the medial and lateral orbital sulci, but sparing any regions of anterior insula at the posterior part of the ablation. The white matter surrounding the corpus striatum and the striatum itself was to be left intact.

Unilateral inferior temporal cortex ablation. The intended extent of the unilateral inferotemporal ablations is identical to such lesions in previous experiments from this laboratory on memory of monkeys after crossed unilateral lesions of frontal and inferotemporal cortex (Parker and Gaffan, 1998b; Browning et al., 2005). Cortex of the right temporal lobe was removed extending from the fundus of the superior temporal sulcus to the fundus of the rhinal sulcus. The posterior part of the lesion included both banks of the occipitotemporal sulcus. The posterior limit of the lesion was a line perpendicular to the superior temporal sulcus, $5 \mathrm{~mm}$ anterior to the inferior occipital sulcus. The anterior limit of the lesion was bounded by a line drawn from the anterior tip of the superior temporal sulcus around the temporal pole to the tip of the rhinal sulcus. All of the cortex was removed within these limits, including both banks of the anterior and posterior middle temporal sulci.

Cortical tissue was removed by subpial aspiration using a small-gauge sucker insulated everywhere except at the tip; electrocautery was applied to remove the pia mater and control bleeding encountered during the ablation.

\section{Histology}

At the completion of all behavioral testing, operated monkeys were deeply anesthetized and transcardially perfused with physiological saline followed by $10 \%$ Formalin. The brains were blocked in the coronal stereotaxic plane posterior to the lunate sulcus, removed from the skull, and allowed to sink in sucrose-Formalin solution. The brains were cut in 50 $\mu \mathrm{m}$ sections on a freezing microtome. Every fifth section was retained and stained with cresyl violet. Figures 3 and 4 illustrate the lesions in experiments 1 and 2, respectively. In all cases, the extents of the lesions were as intended (Fig. 1) and as described in surgical procedures above.

\section{Statistical analysis}

In both of the experiments presented here, the performance is measured as the mean errors per trial for each group across the first 40 trials per problem since the reversal, in which trial 1 is the first trial in which the monkey encounters reversed contingencies on that problem. This ensured comparable analyses between tasks: in experiment 2 , because there was only one problem, the first 40 trials per problem is in fact the first 40 trials since the reversal. An analysis of the errors to criterion in the two tasks was not so directly comparable, but it reveals the same results between the two groups as the analysis of the first 40 trials. In all cases, the data from the error rates were analyzed with one-way ANOVAs with the between-subjects factor of group. 


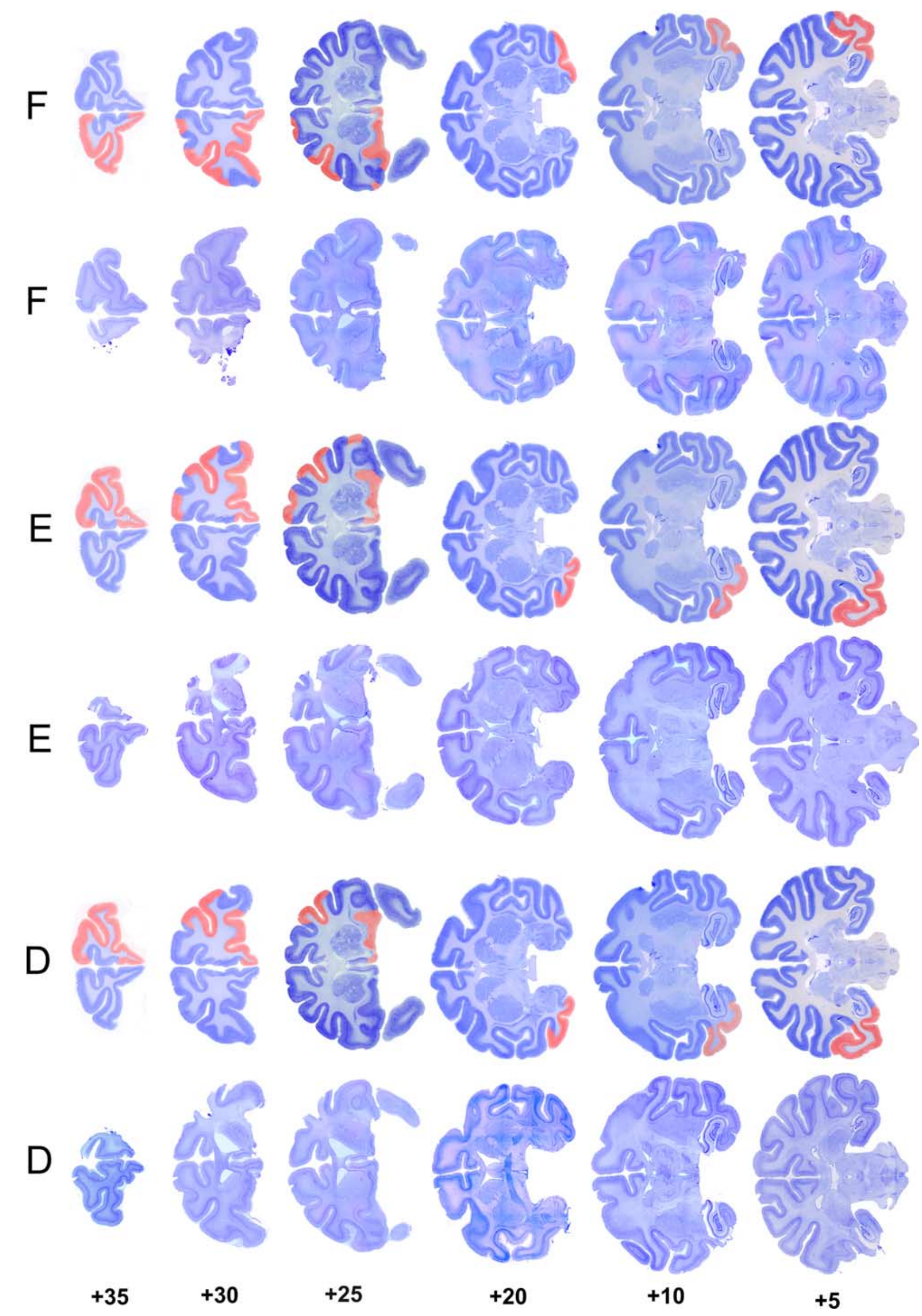




\section{Results}

Experiment 1

In experiment 1 , concurrent reversal learning, monkeys learned 10 concurrent object discriminations in an initial acquisition stage. They subsequently performed two reversal stages, R1 and R2, and, in each of these, half of the 10 problems had their reward contingencies reversed, whereas the other half did not. They were therefore performing reversal learning in the context of concurrent learning about visual objects. The errors made for the stimuli that were reversed at each stage were analyzed, so as to provide a learning curve for reversal learning in the context of concurrent learning. Figure $5 d$ shows the initial learning of the reversed stimuli over 40 blocks by the two groups, collapsed over stages R1 and R2. The performance is measured as the mean errors per trial for each group, plotted across the first 40 trials per problem since the reversal, in which trial 1 is the first trial in which the monkey encounters reversed contingencies on that problem. Forty trials per problem is the minimum number of trials (400 in total, as there are 10 problems) that the monkey had to complete to reach criterion at each stage, and, in most cases, the monkeys attained criterion at this point. To ensure comparable analyses, performance on the first 40 trials of each problem after its reversal was analyzed throughout this study. Analysis of the error rates within these first 40 trials by one-way ANOVA confirms the observation that there are no differences between the two groups $\left(F_{(1,4)}=0.006\right.$, $p=0.941)$. An additional analysis of the errors to criterion measures in all three stages of the task confirmed that there were no differences between the two groups and also showed no effect of stage (in all cases, $F<1$ ), and so there were no differences between stages A, $\mathrm{R} 1$, and R2. As such, it is clear that disconnection of prefrontal cortex from inferior temporal cortex by crossed unilateral lesions of those structures does not impair concurrent reversal learning. This critical result implies that, whatever the role of prefrontal cortex in reversal learning, it is not to provide specific information about specific visual objects processed in IT.

\section{$\leftarrow$}

Figure 4. Coronal sections of the actual and reconstructed lesions of monkeys in experiment 2. This figure should be viewed in landscape format, with the two columns marked D to the left of the page. When viewed in this way, the leftmost column shows actual sections at different anteroposterior levels taken from monkey $D$. In the adjacent column, the extent of the cortical removal is reconstructed and shown in red on cresyl violet-stained sections taken from a normal macaque brain. The remaining columns show similar sections and reconstructions for monkeys $E$ and $F$. This method of displaying the histology better illustrates the size of the lesions, in particular the removed sulci that may not be obvious from the original sections because of collapse of overlying cortex. The numbers to the left indicate millimeters anterior to the interaural line (estimated from Paxinos et al., 2000).
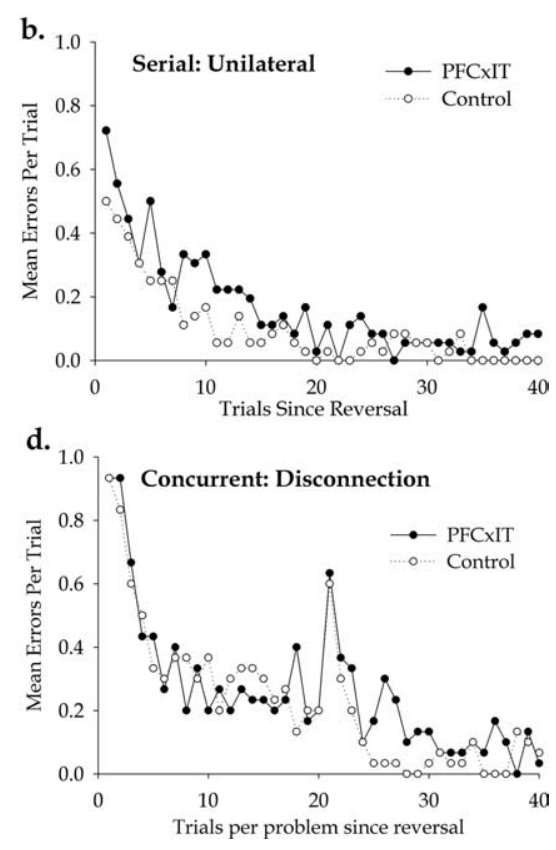

Figure 5. Performance of the monkeys in experiments 1 and 2. $\boldsymbol{a}$, Preoperative performance test in serial reversal learning, experiment 2. The graph shows the mean number of errors committed per trial for the first 40 trials after a reversal (trial 1 being the new reward contingencies are encountered) by the two groups, in a performance test that comprised the prefrontal ablations in serial reversal learning, experiment 2. This performance test comprised the 12 reversals completed after the initial surgery to ablate the prefrontal cortex unilaterally but before the second surgery on the opposite inferior temporal cortex. Performance of the two groups is very similar, suggesting that the unilateral prefrontal lesions had no effect on performance of the task. c, Performance test after disconnection of prefrontal from inferior temporal cortex in serial reversal learning, experiment 2. across the first 40 trials since the reversal. Because there is only one problem in this task, this is therefore equivalent to the trials per

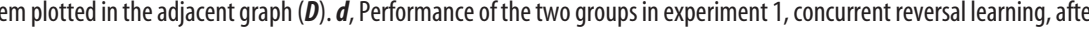
had reversed object reward contingencies relative to the previous stage, collapsed over stages R1 and R2. The performance is and hence the spike reflects a mild decrement at the start of the second session.

\section{Experiment 2}

In experiment 2, the task was a classical serial reversal learning paradigm. Monkeys learned a single visual object discrimination problem to criterion and then learned the same problem with repeatedly reversed reward contingencies over a series of subsequent reversal stages. Both groups of monkeys underwent preoperative training on the task until they had acquired a reversal learning set for the task and were performing at a consistently high level (Fig. 6). Monkeys subsequently performed three performance tests on the task, one before surgery, one after the unilateral prefrontal ablation, and one after the completion of the PFCxIT disconnection. The unoperated control monkeys also performed performance tests after equivalent breaks from testing to the surgical group. The data at these three stages are presented in Figure 5, $a, b$, and $c$, respectively. Each of these graphs is directly comparable with Figure $5 d$, showing learning curves for the two groups measured in mean errors per trial, and plotted across the first 40 trials since the reversal. Because there is only one problem in the task of experiment 2, this measure is therefore equivalent to the trials per problem plotted in Figure $5 d$.

Figure $5 a$ shows the results of the performance test that comprised the last 12 reversals completed before surgery. Analysis of error rates using a one-way ANOVA showed that there were no 


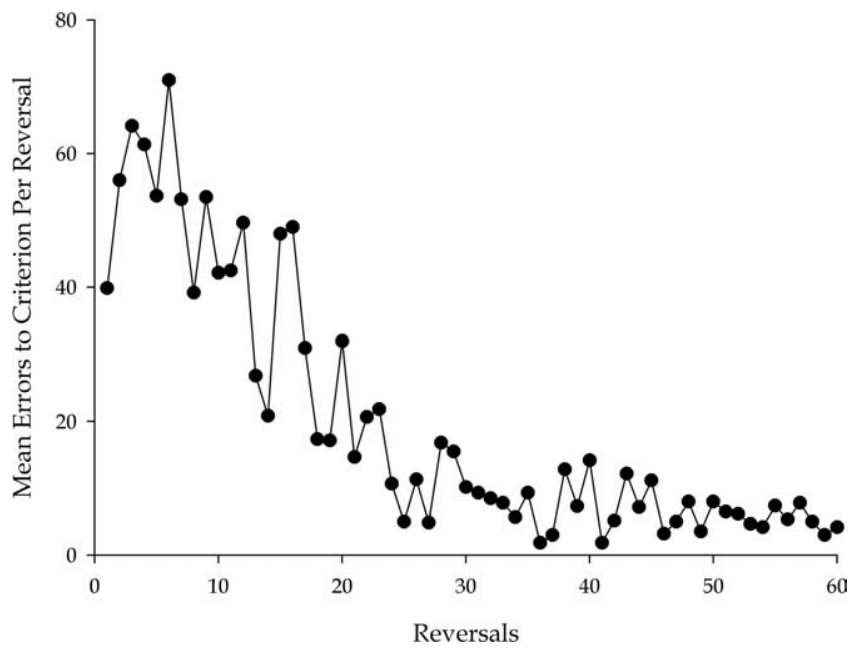

Figure 6. Preoperative formation of a reversal learning set by all six monkeys in experiment 2. The graph shows the mean number of errors made by the six monkeys in reaching criterion at each of the minimum of 60 reversals learned before surgery. The characteristic improvement to a high and stable level of performance indicates the formation of a reversal learning set.

differences between the two groups at this preoperative stage $\left(F_{(1,4)}=5.982, p=0.071\right)$. Figure $5 b$ shows the results of the performance test that comprised the 12 reversals completed after the initial surgery to ablate the prefrontal cortex unilaterally but before the second surgery on the opposite inferior temporal cortex. Similar analysis of error rates using a one-way ANOVA showed that, despite the lesions, there were no differences between the two groups at this stage $\left(F_{(1,4)}=3.115, p=0.152\right)$. As such, the unilateral prefrontal lesions had no effect on performance of the task.

Figure $5 c$ shows the performance of the two groups after the second surgery of the operated group, which completed the PFCxIT disconnection. At this stage, the monkeys had equivalent lesions to those whose data are presented in Figure $5 d$. Here, in contrast to both Figure $5 d$ and the two previous stages above, there was a clear difference between the groups, and analysis of the error rates by one-way ANOVA confirmed a significant effect of group $\left(F_{(1,4)}=23.475, p=0.008\right)$. The comparison of Figure $5 c$ with $a$ and $b$ demonstrates that this difference only appears once the disconnection has been completed. Furthermore, we performed a repeated-measures analysis, to include the marginal but not significant baseline group effects described above in a single analysis, with stage as a within-subjects factor with three levels and group as a between-subjects factor. As well as a significant within subjects effect of stage $\left(F_{(2,8)}=17.822\right.$, Huynh-Feldt test, $p=0.001)$ and between-subjects effect of group $\left(F_{(1,4)}=\right.$ 23.229, $p=0.009$ ), this analysis revealed, as expected, a highly significant group by stage interaction $\left(F_{(2,8)}=14.459\right.$, HuynhFeldt test, $p=0.002$ ). Analyses of errors to criterion provide the same results in all cases. As such, it is clear that, in monkeys with a previously acquired reversal learning set, PFCxIT does cause an impairment in the serial reversal learning task, just as bilateral prefrontal lesions have been repeatedly shown to do (Butter, 1969).

\section{Discussion}

Monkeys with PFCxIT disconnection were unimpaired at a concurrent visual reversal learning task relative to controls. A separate group of monkeys were severely impaired at a serial visual reversal learning task in which they had preoperatively acquired a robust learning set, again relative to controls. Experiments 1 and 2 therefore provide a striking contrast between the effects of PF$\mathrm{C}-\mathrm{IT}$ disconnection in two tasks that both test reversal learning. Despite the clear differences between these tasks, most notably the preoperative training in experiment 2 , the procedure on any one trial is identical in the two tasks, and both tasks require the monkey to inhibit previously learned associations between object and reward. Furthermore, the impairment is seen in the serial reversal task, which only contained one problem and in which the monkeys received extensive preoperative training but is not seen in the concurrent reversal task, which lacked preoperative training and contained a number of problems. Preoperative training in the serial task, and the presence of only one problem in that task, might be expected to make the task easier for the monkeys compared with the concurrent task, yet it was the serial task that was impaired.

In principle, one might attribute the unimpaired performance of the concurrent reversal task to postoperative recovery of function, because the animals in that task were tested one year postoperatively, having performed other experiments in the interval. However, this is not likely to be the explanation of their intact performance in the present experiment, because, at the end of their previous experiment, they still showed a significant and substantial impairment in the visual compound concurrent learning task that was used in that experiment and their performance on that task provided no evidence at all of recovery of function (Browning and Gaffan, 2008). Thus, the surprising lack of impairment we have seen in the concurrent reversal task, contrasting with the substantial impairment in the serial reversal task, cannot be simply explained by either the relative difficulty of the two tasks or the intervals between surgery and testing.

Monkeys with PFC-IT disconnection are severely impaired in visual delayed matching-to-sample (Parker and Gaffan, 1998a; Bussey et al., 2002), visuomotor conditional learning and retention (Bussey et al., 2002), reward-visual conditional learning (Parker and Gaffan, 1998b), a strategy implementation task in which different classes of objects require different patterns of responding (Gaffan et al., 2002), and episodic-like scene learning (Browning et al., 2005; Wilson et al., 2007). Each of these tasks requires the processing of temporally complex visual events, in contrast to concurrent object-reward association learning (Parker and Gaffan, 1998b; Gaffan et al., 2002), which does not. Browning and colleagues (Browning et al., 2005, 2007; Browning and Gaffan, 2008) have therefore proposed that PFC has a specific role in the representation of temporally complex events and that disconnection of PFC from IT will therefore impair performance of any task that requires the processing of temporally complex events that include at least one piece of visual object information. We contend that this theory provides the clearest explanation for the striking dissociation of impairments presented here.

In many traditional learning tasks used with macaque monkeys, the monkey has to learn to associate an object with a subsequently presented reward. This is a temporally simple, two-term association between one event (the object) and the immediately following event (the reward). If, however, the association that the monkey has to learn is more complex, involving three or more terms, the elements of which are spread over a period of time, the event can be said to be temporally complex. According to the view of learning set formation suggested by previous experiments (Murray and Gaffan, 2006; Browning et al., 2007), experiment 2 involves the processing of temporally complex visual events because of the learning set acquired preoperatively (Fig. 6). We know that the reversal learning set is the same ability as the dis- 
crimination learning set studied by Browning et al. (2007) because there is excellent transfer between the two (Schrier, 1966). The fact that monkeys are able to represent serially presented stimuli in a configural way, as shown by the presence of mediated generalization, supports the idea that initial learning set acquisition benefits from temporal contiguity of the problems involved (Gaffan and Dickinson, 2007). In contrast, tasks in which problems are learned concurrently do not promote the acquisition of a learning set because successive examples of individual problems are too far apart for the formation of the prospective memory that appears to underlie the learning set (Murray and Gaffan, 2006). Experiment 1 requires concurrent learning and therefore does not promote the formation of a reversal learning set.

In many cases, the temporally complex event proposal is compatible with extant theories of PFC function along the lines of executive cognitive control (Miller and Cohen, 2001; O'Reilly et al., 2002), behavioral inhibition (Aron et al., 2004), or general intelligence (Duncan et al., 2000). However, because they make fairly generalized predictions about the way in which PFC acts on posterior regions of cortex, these major theories are unable to account for the very significant difference between the two very similar tasks in the current experiments. It is possible that one may want to describe the processing of temporally complex events as an example of an executive function. Executive function is a term without a fixed definition in the literature, and, although it is no doubt of great use in some situations, it is difficult to generate specific and testable predictions about PFC function from the statement that it is related to executive function. The temporally complex event approach, in contrast, generates a number of specific and testable hypotheses, one of which has been clearly supported here, and therefore it is a preferable description for PFC function, at least in the domain of visual learning. Whether this role extends beyond this domain remains an open question and one that will be the subject of future studies.

The current results question the mode of interaction of PFC with other regions of the brain in cognitive control. They contradict any account of the mechanism of reversal learning that requires PFC to actively inhibit stimulus representations in IT. Other accounts suggest that PFC may instead enhance representations of task-relevant stimuli (Miller and Cohen, 2001; Egner and Hirsch, 2005), for example in IT (O’Reilly et al., 2002), but this possibility is also ruled out by the current study, at least with respect to representations of visual stimuli in IT. The PFC may exert cognitive control without affecting stimulus representations, via direct interactions with, for example, premotor cortical regions or corticostriatal circuits. Such interactions would be retained after PFCxIT, yet the animals in experiment 2 still exhibit significant impairment.

Schoenbaum et al. (1999) have studied the specific interaction between orbital frontal cortex (OFC) and the amygdala in the context of reversal learning. They argue that OFC does not have a general role in inhibiting responses (Schoenbaum et al., 2002), in line with our findings here, and show counter-intuitively that amygdala lesions abolish OFC-dependent reversal impairments (Stalnaker et al., 2007) (see also Baxter and Browning, 2007). The relative role of the amygdala or the OFC-amygdala interaction in serial and concurrent reversal learning remains an open question, but it should be noted that bilateral excitotoxic lesions of the amygdala do not impair serial reversal learning (Izquierdo and Murray, 2007).

One might attempt to explain the current results in terms of working memory, arguing that the retention of temporally complex events is an example of working memory in action. For example, perhaps serial reversal learning involves the use of working memory representations for the correct stimuli, and these representations are maintained to support correct responding from one trial to the next and can bias the associated object representations in IT. This would contrast with concurrent reversal learning, in which multiple items would have to be stored concurrently in working memory, to the extent that the task requirement would exceed a monkey's working memory capacity. Furthermore, in the concurrent task, the extended delay between repeated presentations of the same stimulus might lead to decay of the working memory for that stimulus.

We regard the temporally complex event hypothesis as a more specific and testable description of the function of the prefrontalinferotemporal interaction than working memory, because it specifically describes a type of representation in PFC rather than a general cognitive process. Most importantly, recent data from our laboratory directly contrasts a temporally complex visual memory with a temporally simple visual working memory, showing that PFCxIT impairs only memory for the former (Browning and Gaffan, 2008). Although a description in terms of working memory is possible in the cases of some of the impairments produced by PFCxIT, it is not a generally applicable explanation of those results.

The ability to process temporally complex events is vital to a wide range of behaviors, and indeed we argue that this is the reason for the wide-ranging impairments after bilateral lesions of PFC. This, of course, means that many impairments noted in support of the theories of executive function and behavioral inhibition will also support the notion that PFC specifically processes temporally complex events. We believe that the current data, however, very clearly disambiguate these theories and provide robust support for the latter view.

\section{References}

Aron AR, Robbins TW, Poldrack RA (2004) Inhibition and the right inferior frontal cortex. Trends Cogn Sci 8:170-177.

Baxter MG, Browning PG (2007) Two wrongs make a right: deficits in reversal learning after orbitofrontal damage are improved by amygdala ablation. Neuron 54:1-3.

Browning PG, Gaffan D (2008) Prefrontal cortex function in the representation of temporally complex events. J Neurosci 28:3934-3940.

Browning PG, Easton A, Buckley MJ, Gaffan D (2005) The role of prefrontal cortex in object-in-place learning in monkeys. Eur J Neurosci 22:3281-3291.

Browning PGF, Easton A, Gaffan D (2007) Frontal-temporal disconnection abolishes object discrimination learning set in macaque monkeys. Cereb Cortex 17:859-864.

Brutkowski S (1965) Functions of prefrontal cortex in animals. Physiol Rev 45:721-746.

Bussey TJ, Wise SP, Murray EA (2002) Interaction of ventral and orbital prefrontal cortex with inferotemporal cortex in conditional visuomotor learning. Behav Neurosci 116:703-715.

Butter CM (1969) Perseveration in extinction and in discrimination reversal tasks following selective frontal ablations in Macaca mulatta. Physiol Behav 4:163-171.

Clarke HF, Dalley JW, Crofts HS, Robbins TW, Roberts AC (2004) Cognitive inflexibility after prefrontal serotonin depletion. Science 304:878-880.

Dias R, Robbins TW, Roberts AC (1997) Dissociable forms of inhibitory control within prefrontal cortex with an analog of the Wisconsin Card Sort Test: restriction to novel situations and independence from "online" processing. J Neurosci 17:9285-9297.

Duncan J, Seltz RJ, Kolodny J, Bor D, Herzog H, Ahmed A, Newell FN, Emslie $\mathrm{H}$ (2000) A neural basis for general intelligence. Am J Ophthalmol 130:687.

Eacott MJ, Gaffan D (1991) The role of monkey inferior parietal cortex in visual discrimination of identity and orientation of shapes. Behav Brain Res 46:95-98. 
Egner T, Hirsch J (2005) Cognitive control mechanisms resolve conflict through cortical amplification of task-relevant information. Nat Neurosci 8:1784-1790.

Fellows LK, Farah MJ (2003) Ventromedial frontal cortex mediates affective shifting in humans: evidence from a reversal learning paradigm. Brain 126:1830-1837.

Gaffan D (1994) Dissociated effects of perirhinal cortex ablation, fornix transection and amygdalectomy: evidence for multiple memory systems in the primate temporal lobe. Exp Brain Res 99:411-422.

Gaffan D, Dickinson A (2007) Mediated generalization in discrimination learning by rhesus monkeys. Q J Exp Psychol 61:558-568.

Gaffan D, Harrison S, Gaffan EA (1986) Visual identification following inferotemporal ablation in the monkey. Q J Exp Psychol B 38:5-30.

Gaffan D, Easton A, Parker A (2002) Interaction of inferior temporal cortex with frontal cortex and basal forebrain: double dissociation in strategy implementation and associative learning. J Neurosci 22:7288-7296.

Gross CG (1978) Inferior temporal lesions do not impair discrimination of rotated patterns in monkeys. J Comp Physiol Psychol 92:1095-1109.

Iversen SD, Mishkin M (1970) Perseverative interference in monkeys following selective lesions of the inferior prefrontal convexity. Exp Brain Res 11:376-386.

Izquierdo A, Murray EA (2007) Selective bilateral amygdala lesions in rhesus monkeys fail to disrupt object reversal learning. J Neurosci 27:1054-1062.

Izquierdo A, Suda RK, Murray EA (2004) Bilateral orbital prefrontal cortex lesions in rhesus monkeys disrupt choices guided by both reward value and reward contingency. J Neurosci 24:7540-7548.

Jones EG, Powell TP (1970) An anatomical study of converging sensory pathways within the cerebral cortex of the monkey. Brain 93:793-820.

Konishi S, Nakajima K, Uchida I, Kikyo H, Kameyama M, Miyashita Y (1999) Common inhibitory mechanism in human inferior prefrontal cortex revealed by event-related functional MRI. Brain 122:981-991.

Kuypers HG, Szwarcbart MK, Mishkin M, Rosvold HE (1965) Occipitotemporal corticocortical connections in the rhesus monkey. Exp Neurol 11:245-262.

Miller EK, Cohen JD (2001) An integrative theory of prefrontal cortex function. Annu Rev Neurosci 24:167-202.

Milner B (1963) Effects of different brain lesions on card sorting. Arch Neurol 9:90.
Mitchell AS, Gaffan D (2008) The magnocellular mediodorsal thalamus is necessary for memory acquisition, but not retrieval. J Neurosci 28:258-263.

Murray EA, Gaffan D (2006) Prospective memory in the formation of learning sets by rhesus monkeys (Macaca mulatta). J Exp Psychol Anim Behav Process 32:87-90.

Nakahara K, Hayashi T, Konishi S, Miyashita Y (2002) Functional MRI of macaque monkeys performing a cognitive set-shifting task. Science 295:1532-1536.

O'Reilly RC, Noelle DC, Braver TS, Cohen JD (2002) Prefrontal cortex and dynamic categorization tasks: representational organization and neuromodulatory control. Cereb Cortex 12:246-257.

Parker A, Gaffan D (1998a) Interaction of frontal and perirhinal cortices in visual object recognition memory in monkeys. Eur J Neurosci 10:3044-3057.

Parker A, Gaffan D (1998b) Memory after frontal/temporal disconnection in monkeys: conditional and non-conditional tasks, unilateral and bilateral frontal lesions. Neuropsychologia 36:259-271.

Paxinos G, Huang X-F, Toga AW (2000) The rhesus monkey brain in stereotaxic coordinates. San Diego: Academic.

Rolls ET, Hornak J, Wade D, McGrath J (1994) Emotion-related learning in patients with social and emotional changes associated with frontal lobe damage. J Neurol Neurosurg Psychiatry 57:1518-1524.

Schoenbaum G, Chiba AA, Gallagher M (1999) Neural encoding in orbitofrontal cortex and basolateral amygdala during olfactory discrimination learning. J Neurosci 19:1876-1884.

Schoenbaum G, Nugent SL, Saddoris MP, Setlow B (2002) Orbitofrontal lesions in rats impair reversal but not acquisition of go, no-go odor discriminations. NeuroReport 13:885-890.

Schrier AM (1966) Transfer by macaque monkeys between learning-set and repeated-reversal tasks. Percept Motor Skills 23:787-792.

Stalnaker TA, Franz TM, Singh T, Schoenbaum G (2007) Basolateral amygdala lesions abolish orbitofrontal-dependent reversal impairments. Neuron 54:51-58.

Wilson CR, Gaffan D, Mitchell AS, Baxter MG (2007) Neurotoxic lesions of ventrolateral prefrontal cortex impair object-in-place scene memory. Eur J Neurosci 25:2514-2522. 